\section{Is it safe to discharge treated proliferative diabetic retinopathy patients from the hospital eye service to a community screening programme?}

GS Negretti and GC Vafidis
Central Eye Services, Central Middlesex Hospital, North West London Hospitals NHS Trust, London, UK

Correspondence: GS Negretti, Moorfields Eye Hospital NHS Foundation Trust, 162 City Road, London EC1V 2PD, UK Tel: +44207 2533411; Fax: + 442075662972 E-mail: guynegretti@ gmail.com

Received: 9 September 2013

Accepted in revised form: 26 January 2014

Published online:

14 March 2014

This work has previously been presented as a poster at the ARVO annual meeting 2013.

\begin{abstract}
Purpose To investigate the distribution of new vessels $(\mathrm{NV})$ in patients treated with pan-retinal photocoagulation for proliferative diabetic retinopathy (PDR). To assess whether it is safe to discharge treated PDR patients to the NHS Diabetic Eye Screening Programme (DESP) which uses two mydriatic $45^{\circ}$ fields of each eye.

Methods Consecutive treated PDR patients undergoing fundus fluorescein angiography between July 2010 and October 2011 for the purpose of looking for NV were included. The distribution of NV was mapped. In particular it was noted whether NV occurred in the area covered by the DESP standard screening images.

Results A total of 76 patients (108 eyes) met the inclusion criteria for the study. Leaking NV were found inside the DESP fields in $89 \%$ of study patients. In 108 eyes with leaking NV, there were a total of $35 \mathrm{NVD}$ and 336 NVE. NV were found within DESP fields in $83 \%$ of eyes. Of the $336 \mathrm{NVE}, 54 \%$ occurred within and $46 \%$ outside DESP standard fields. There was no statistically significant difference in the retinal quadrant distribution of NVE.

Conclusions If these findings apply to the whole treated PDR population, NVE would be identified in $89 \%$ of patients undergoing DESP screening. This would support stable treated PDR patients being monitored within the DESP. We found no preferential clustering of NV within quadrants or between posterior and less posterior retina suggesting that there would be no benefit to
\end{abstract}

the DESP of taking an additional field or graders concentrating on one particular quadrant more than another.

Eye (2014) 28, 696-700; doi:10.1038/eye.2014.48; published online 14 March 2014

\section{Introduction}

Diabetic retinopathy remains a major cause of blindness in working age people worldwide. ${ }^{1}$ As disease prevalence rises, ${ }^{2}$ prevention of vision loss due to diabetes becomes increasingly important. An essential first step, as outlined by The St Vincent Declaration of 1989, is the introduction of systematic screening for diabetic retinopathy. ${ }^{3}$

Between 2000 and 2002, a population-based diabetic retinopathy screening programme was proposed for the four nations of the United Kingdom. ${ }^{4-6}$ In 2006, the National Diabetic Eye Screening Programme was implemented in England (NHS DESP) with the aim of reducing blindness due to diabetic retinopathy. In this programme every diabetic person over 12 years of age registered with a GP in England is invited for annual digital retinal photography. In 20112012, NHS DESP identified 2.59 million people with diabetes eligible for screening, $91 \%$ (2.36 million) were invited for digital retinal photography and 1.9 million accepted; a screening uptake of $81 \%$. $^{7}$

In the programme, standard images-two mydriatic $45^{\circ}$ fields of each eye, one centred on disc, one on macula-are graded by a quality assured team. If sight-threatening diabetic retinopathy is identified, the patient is referred 
to the hospital eye service (HES). Following completion of treatment, stable patients are referred from HES back to the screening programme for annual digital photography. In 2012, a new common pathway was introduced to standardise patient pathways across NHS DESP. This includes a new grade 'stable treated proliferative diabetic retinopathy' (NSC grade R3s) ${ }^{7}$ and describes eyes that have full pan-retinal photocoagulation (PRP) treatment for proliferative retinopathy and are discharged from HES to be monitored in NHS DESP. If significant changes to baseline discharge photographs occur, R3s eyes are to be re-referred to HES as R3a (active R3). Significant change is described as 'signs of active neovascularisation including active new vessels, pre-retinal or vitreous haemorrhage' ${ }^{7}$ The activity status of persistent new vessels depends on clinical assessment and it is acknowledged by DESP that although not all R3s re-referral decisions may be clinically urgent, this simple approach avoids delayed referral of R3 retinopathy.

In this study our aim was to identify sites of new or re-activated NV in previously treated proliferative (R3s) eyes and whether they would be present within the boundaries of NHS DESP standard fields.

\section{Materials and methods}

This was a single-centre retrospective cohort study. Study eyes were recruited from the eye clinic fundus fluorescein angiogram (FFA) log. We identified consecutive patients who had undergone FFA to look for leaking new vessels in treated proliferative diabetic retinopathy between July 2010 and October 2011 (16 months) as part of their subsequent management in HES. Serial colour fundus photographs and clinical examinations in HES were the basis for suspected activity of new vessels in these patients and the investigated R3s eyes were subsequently analysed for this study.

FFA was performed after injection of $5 \mathrm{ml}$ of $20 \%$ sodium fluorescein using a $50^{\circ}$ Zeiss $450^{\text {PLUS }}$ IR camera (Oberkochen, Germany). Our FFA protocol in diabetic eyes consists of an eight-field survey of seven standard DRS fields ${ }^{8}$ including disc-centred (field 1) and macularcentred (field 3 ) images, with an additional peripheral image nasal to the disc.

Interpretation of FFA images was on the basis of characteristics described in ETDRS. ${ }^{9}$ A discrete patch of new vessels elsewhere (NVE) was characterised as a continuous area of diffuse fluorescein leakage surrounded by retina without leakage in the mid-venous phase of the angiogram and continuing into the late phases indicating the presence of new vessels. Sites of discrete NVE patches were mapped on to a retinal template. The $50^{\circ} \mathrm{FFA}$ fields of images 1 and 3 were larger than the corresponding DESP standard images and a $45^{\circ}$ circle was therefore mapped on to the centre of each $50^{\circ}$ image to approximate to the DESP fields. The position of NVE patches within or outside these fields was noted.

Study exclusions were:

1. Eyes with known additional retinovascular pathology, for example, artery or vein occlusion, sickle cell retinopathy.

2. Eyes with significant media opacity (eg, cataract) preventing accurate analysis.

3. Eyes in which the FFA omitted one or more of the retinal survey fields.

4. Eyes that did not have full PRP treatment (ie, where one or more disc areas of eligible retina had no evidence of laser scatter).

Statistical analysis was performed using Microsoft Excel with analysis of variance performed using Statplus (AnalystSoft Inc., Alexandria, VA, USA). Statistical significance was defined as $P<0.05$. The study was registered in the trust audit register.

\section{Results}

\section{Patients}

During the 16 months of the study, 116 patients with treated PDR and suspected active new vessels had FFA to look for leaking NV. New vessels were detected in one or both eyes in $72 \%(84 / 116)$ of these. Of 84 patients with $\mathrm{NV}, 76$ met the study inclusion criteria (108 eyes) and these were analysed further (see Figure 1). Study patients' mean age was 64 years (range 45-85 years) with more males than females ( $43 \mathrm{~m}: 33 \mathrm{f}$ ).

Leaking new vessels were found inside the disccentred and macular-centred DESP fields in $89 \%$ study patients $(68 / 76)$. If these patients had been photographed using standard DESP colour images, $11 \pm 7 \%$ (95\% CI) would have had all NV outside screened fields. This gives a sensitivity of $89 \%$ for DESP fields in our cohort of R3s patients with documented NV.

Using the macular-centred image alone, 63\% (48/76) patients had NV imaged within the screening field. This means that in $37 \pm 7 \%(95 \% \mathrm{CI})$ patients, all NV were outside the imaged retina. This gives a sensitivity of $63 \%$ for the macula-only field to image NV in R3s patients.

\section{Eyes}

In 108 eyes with leaking new vessels there were 35 NVD and 336 NVE. New vessels were found within DESP fields in 90 eyes (83\%). In 52 eyes (48\%), all NV were 


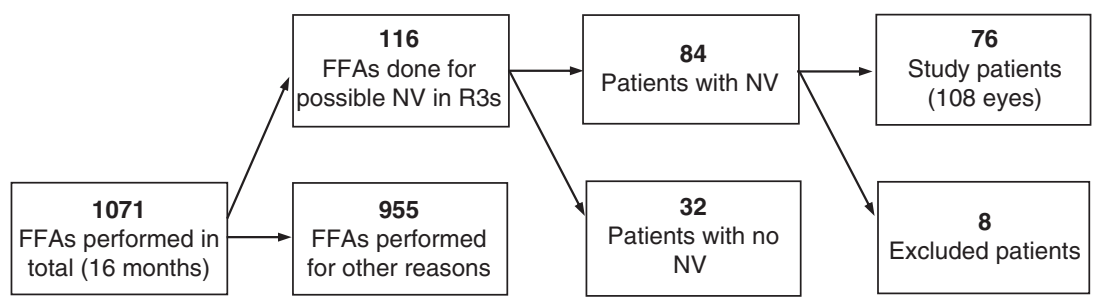

Figure 1 A flow-chart showing how the final number of patients in the study arose.

within, and in 18 (17\%), all NV were outside DESP fields. In the remaining 38 eyes (35\%) NV were found both within and outside DESP fields. In seven eyes, nine patches of NVE were imaged within the $50^{\circ} \mathrm{FFA}$ field but outside the $45^{\circ}$ DESP field superimposed on images 1 and 3.

\section{Retinal quadrant distribution of NVE}

Of 336 NVE patches, 54\% (183) occurred within and 46\% (153) outside DESP standard fields. The difference between central vs peripheral distribution was not significant ( $P=0.3$ unpaired $t$-test).

Retinal quadrant distribution of NVE also showed no significant differences with 30\% (102) inferotemporal, $23 \%$ (76) superotemporal, 26\% (86) inferonasal, and 21\% (72) superonasal ( $P=0.26$ analysis of variance) (see Figure 2). There was no significant difference in distribution of $\mathrm{NV}$ between quadrants either for central $45^{\circ}$ images $(P=0.19)$ or for peripheral images $(P=0.66)$.

\section{Discussion}

We found that $89 \%$ of treated PDR patients with leaking new vessels on FFA had some of these vessels within NHS DESP standard fields. Thus in the R3s population where new vessels may be clinically active, almost 9 of 10 patients had at least some of their leaking new vessels within the two retinal photographic fields that would be available to DESP graders to detect change when compared with the baseline discharge image set. The potential to detect new vessel changes in R3s with $89 \%$ sensitivity would be acceptable in the context of population screening tests, for example, in mammography for breast cancer screening, there is an overall sensitivity of approximately $79 \% .^{10}$

Leakage of fluorescein from new vessels on retinal angiography is not reported as a diagnostic feature of untreated proliferative disease in the landmark treatment studies although both used FFA at baseline and followup visits. 8 , 9 However FFA has been used in subsequent publications to indicate persistent leakage from new vessels in eyes with previous laser therapy. ${ }^{11,12}$ In this



Figure 2 Representation of a fundus showing NVE distribution between quadrants, both inside and outside the standard DESP fields. Percentages of NVE are shown in brackets. IN, inferonasal; IT, inferotemporal; SN, superonasal; ST, superotemporal.

study we have also used it as a marker for sites of new or persistent NV. We recognise that although not all new vessels that leak fluorescein will require further laser, treated new vessels that do not leak fluorescein are probably stable and have not been identified in our study as NV.

Furthermore, although this study found leaking vessels within the DESP imaged retina in $89 \%$ patients and $83 \%$ eyes, these posterior leaking vessels made up only $54 \%$ NVE that we identified in these eyes. In addition, we cannot speculate on how many more NV lie outside the eight-field imaging used here. A recent publication describing ultra-wide-field imaging demonstrates 3.2 times more retinal surface area than seven-field standard imaging ${ }^{13}$ and this may be evaluated for retinal screening programmes in the future.

When we used a single $45^{\circ}$ macula-centred field for monitoring R3s, we found a sensitivity of only $63 \%$ for imaging the sites of leaking NV. This is of relevance to the Scottish Diabetic Retinopathy Screening Programme 
where only one $45^{\circ}$ macula-centred image is used for DR screening. ${ }^{14}$

There is a long running debate about the number of fields required for safe screening. ${ }^{15}$ With an extended role of screening programmes in monitoring treated disease, there is a lack of evidence to support the number and position of chosen fields. We found no preferential clustering of $\mathrm{NV}$ within quadrants or between posterior and less posterior retina in this study. This suggests that there would be no benefit to DESP of taking an additional field to monitor R3s patients nor for graders to concentrate their efforts on one quadrant more than another when analysing images from these patients.

Our study was problematic in that we had to make adjustments for the different field sizes of our FFA and DESP cameras. However it was reassuring that only 9 (2.7\%) NVE fell within the border area between $45^{\circ}$ and $50^{\circ}$ fields. We feel that this makes it unlikely that the exact positioning of the DESP field makes a critical difference to the likelihood of active NV falling within or outside the imaged retina.

All patients in our study underwent FFA because they were suspected of having new vessels after clinical examination in the hospital eye service. Since the posterior pole is usually more accessible to examination than peripheral retina, this may have biased patient selection. However, this parallels the situation in the screening programme, where only posterior retina is photographed. Not all R3s eyes were found to have new vessels, $28 \%$ of patients imaged had no leakage on angiography.

In the modern NHS there is consensus about the need to reduce the number of patients attending hospitals in favour of monitoring in the community. We estimate that the R3s patient population makes up at least $0.5 \%$ of our total diabetic register for DESP locally (22000 in 2012-13) and discharge from HES back to annual or biannual recall within the screening programme would represent cost efficiency for the health service.

Complications associated with active new vessels in diabetic retinopathy are well known. Those with untreated high-risk proliferative diabetic retinopathy have a high risk of severe visual loss. ${ }^{16}$ However the clinical relevance of new vessels that leak fluorescein in treated PDR is unknown. Presumably it is a marker of serum and blood release into the vitreous cavity from NV. The latter may lead to further visual loss from vitreous haemorrhage or traction detachment. It can also indicate the possibility of anterior segment neovascularisation and leakage.

There are few studies looking at the long-term complications of treated PDR. ${ }^{17,18}$ The incidence of vitreous haemorrhage after treatment has been reported as high as $39 \%$ with $20 \%$ blind within 5 years. ${ }^{18}$
With early use of antiVEGF agents, prevention of advanced complications such as rubeotic glaucoma and fibrovascular traction detachment can be controlled even in previously photocoagulated retinas. ${ }^{19}$ It is therefore important to identify these eyes so that they can be clinically assessed and treated as quickly as possible.

\section{Summary}

\section{What was known before}

- A new grade has recently been introduced into the NHS DESP. R3s patients who have stable treated PDR will be monitored within the community screening programme.

- It is unclear whether new vessels occurring in these patients will be picked up by the limited photographic fields of the NHS DESP.

What this study adds

- The majority of treated PDR patients, if they develop new vessels, will be picked up by the NHS DESP with their limited photographic views.

- There appears to be no preferential clustering of NV within quadrants or between posterior and less posterior retina in these patients.

\section{Conflict of interest}

The authors declare no conflict of interest.

\section{References}

1 Resnikoff S, Pascolini D, Etya'ale D, Kocur I, Pararajasegaram R, Pokharel GP et al. Global data on visual impairment in the year 2002. Bull World Health Organ 2004; 82: 844-851.

2 Wild S, Roglic G, Green A, Sicree R, King H. Global prevalence of diabetes: estimates for the year 2000 and projections for 2030. Diabetes Care 2004; 27(5): 1047-1053.

3 The Saint Vincent Declaration on diabetes care and research in Europe. Acta Diabetologia 1989; 10(Suppl): 143-144.

4 Garvican L, Clowes J, Gillow T. Preservation of sight in diabetes: developing a national risk reduction programme. Diabet Med 2000; 17(9): 627-634.

5 Gillow JT, Gray JA. The National Screening Committee review of diabetic retinopathy screening. Eye (Lond) 2001; 15(Pt 1): 1-2.

6 Facey K, Cummins E, Macpherson K, Morris A, Reay L, Slattery J. Organisation of services for diabetic retinopathy screening. Health Technology Assessment Report 1, Health Technology Board for Scotland: Glasgow, Scotland, UK 2002.

7 Diabetic Eye Screening Revised Grading Definitions November 2012, The grading assessment committee of DESP. Available http://diabeticeye.screening.nhs.uk/.

8 Diabetic Retinopathy Study Research Group. Design methods and baseline results. DRS report no. 6. Invest Ophthalmol 1981; 21: 149-209.

9 Classification of Diabetic retinopathy from fluorescein angiograms. ETDRS report number 11. Ophthalmology 1991; 98: $807-822$. 
10 Yankaskas BC, Taplin SH, Ichikawa L, Geller BM, Rosenberg RD, Carney PA et al. Association between mammography timing and measures of screening performance in the United States. Radiology 2005; 234(2): 363-373.

11 Menchini U, Lanzetta P, Soldano F, Ferrari E, Virgili G. Continuous wave Nd:YAG laser photocoagulation in proliferative diabetic retinopathy. Brit J Ophthalmol 1995; 79: 642-645.

12 Muqit MMK, Marcellino GR, Henson DB, Young LB, Turner GS, Stanga PE. Pascal panretinal laser ablation and regression analysis in proliferative diabetic retinopathy: Manchester Pascal Study Report 4. Eye (Lond) 2011; 25(11): 1447-1456.

13 Wessel MM, Aaker GD, Parlitsis G, Cho M, D'Amico DJ, Kiss S. Ultra-wide-field angiography improves the detection and classification of diabetic retinopathy. Retina 2012; 32: 785-791.

14 NHS Scotland National Diabetes Retinopathy Screening. www.ndrs.scot.nhs.uk.
15 Moss SE, Meuer SM, Klein R, Hubbard LD, Brothers RJ, Klein BE. Are seven standard photographic fields necessary for classification of diabetic retinopathy? Invest Ophthalmol Vis Sci 1989; 30(5): 823-828

16 Diabetic Retinopathy Study Research Group. Photocoagulation treatment of proliferative diabetic retinopathy: clinical application of diabetic retinopathy study (DRS) findings. DRS report no. 8. Ophthalmology 1981; 88: 583-600.

17 Kleinmann G, Hauser D, Schechtman E, Landa G, Bukelman A, Pollack A. Vitreous hemorrhage in diabetic eyes previously treated with panretinal photocoagulation. Int Ophthalmol 2008; 28: 29-34.

18 Ajvazi H. Proliferative diabetic retinopathy-results of the microsurgical and laser treatment. Med Arh 2010; 64: 165-167.

19 Schmidinger G, Maar N, Bolz M, Scholda C, SchmidtErfurth U. Repeated intravitreal bevacizumab (Avastin) treatment of persistent new vessels in proliferative diabetic retinopathy after complete panretinal photocoagulation. Acta Ophthalmol 2011; 89(1): 76-81. 